\title{
La problemática historiográfica sobre Jesús y su alcance teológico
}

\author{
Victor Ml. Mora Mesén*
}

Recibido: Julio 2011 • Aceptado: Setiembre 2011

RESUMEN

Este ensayo busca analizar la problemática surgida sobre Jesús histórico. ¿El Jesús de la historia es el mismo Jesús de los evangelios? El tema es polémico y las reconstrucciones serias no lo han agotado. Según el autor, la investigación histórica erudita sobre Jesús es distinta a la visión mediática. Ella tiene que ver con cuestiones literarias, arqueológicas, sociológicas y culturales. De ahí que este trabajo se centre en cuatro subtemas medulares: a. El problema de las fuentes, b. Los criterios para determinar la autenticidad de los datos, c. El problema de la hermenéutica, y d. La utilidad de la investigación para el quehacer cristológico.

Palabras clave: Jesús histórico, problemática historiográfica, los evangelios, el problema hermenéutico, método histórico-crítico, quehacer cristológico.

\section{ABSTRACT}

This essay looks for analyzing the problematic arisen about historic Jesus. Is the Jesus from history the same than the Jesus from the Gospels? The issue is polemic and the real rebuilding has not used up. According to the author of the scholar-historic investigation about Jesus it is different to the media vision. She has to do with literature, archeological, sociological, and cultural issues. Therefore, this work is centered on four main subtopics: a. The problem of the sources, b. The criteria to determine the authenticity of the facts, c. The problem of the hermeneutics, and d. The utility of the research for the human endeavor.

Key words: Jesús histórico, problemática historiográfica, los evangelios, el problema hermenéutico, método histórico-crítico, quehacer cristológico.

* Licenciado en Exégesis Bíblica por el Pontificio Instituto Bíblico de Roma, realizó estudios de Filosofía y Teología en el Instituto Teológico de América Central (ITAC). Ha sido Custodio Provincial de los Frailes Menores Conventuales en Costa Rica, Director Ejecutivo del ITAC y Presidente de la Conferencia de Religiosos de Costa Rica. Actualmente es profesor de Sagrada Escritura en el ITAC y Director General del Saint Francis College. frayvictor@gmail.com 


\section{Introducción}

Se habla mucho del Jesús histórico, sea en obras eruditas, en programas televisivos de amplia divulgación o en obras totalmente fantasiosas y carentes de toda seriedad. Lo cierto es que para el ojo crítico ninguna de las reconstrucciones serias ha dado por agotado el tema ${ }^{1}$. La polémica ha surgido, por otro lado, como un fenómeno mediático que ha puesto en entredicho el andamiaje dogmático tradicional. Con seriedad conceptual y metodológica o sin ella, el Jesús de la historia se enarbola en nuestro tiempo como una bandera ideológica o como un producto con un amplio mercado entre los estratos medios de la población. No es extraño oír comentarios acerca de cómo se ha tergiversado la figura de Jesús por la acción de una jerarquía eclesiástica ávida de poder en la época de Constantino, el emperador romano que utilizó el cristianismo como un soporte a su autoridad. La definición de los textos canónicos del NT tendría como telón de fondo -para esta visión mediática- la intención de traicionar al predicador de Galilea, al cual se quiso silenciar

1. Salvo algunas excepciones como Bermejo, $\mathrm{F}$. (2005). Historiografía, exégesis e ideología. La ficción contemporánea de las "Tres búsquedas" del Jesús Histórico. (F. d. Catalunya, \& F. d., Eds.) $R c a T$, $X X X(2), 349-406$. para usar una figura dogmática funcional a los intereses imperiales en su lugar.

No hay duda de que estas opiniones no dejan de ser una tergiversación de la realidad histórica del contenido de los textos en cuestión y de los procesos de canonización, pero la narración de una conspiración siempre atrae la atención del gran público. La realidad de la investigación histórica erudita sobre Jesús es muy distinta, ya que su problemática tiene que ver con cuestiones literarias, arqueológicas, sociológicas y culturales. Se trata de entender a un personaje real de un contexto distante al nuestro, pero que todavía sigue siendo profundamente significativo para nuestro tiempo. Occidente ha sido marcado por el cristianismo, por lo que la figura de Jesús de Nazaret está a la base de muchos de nuestros valores y concepciones religiosas y políticas. Pero acercarse a su realidad histórica nos abre las puertas de otro mundo: el judaísmo helenista del siglo primero de nuestra era. Para sus contemporáneos, Jesús también significó un desafío y una pregunta: unos lo siguieron apasionadamente, otros decidieron acabar con su vida. Investigar porqué ocurrió esto, es el núcleo básico del estudio histórico-crítico de su persona.

En el presente ensayo intentaremos dar cuenta sintéticamente 
de los principales problemas, alcances y limitaciones que tiene la investigación histórica sobre Jesús. Para ello dividiremos la temática en cuatro temas medulares: el problema de las fuentes que contienen información histórica fidedigna, los criterios para determinar la autenticidad de los datos, el problema hermenéutico implicado en la reconstrucción histórica y la utilidad de esta investigación para el quehacer cristológico.

\section{El problema de las fuentes para el conocimiento histórico de Jesús}

Cualquier investigación histórica debe definir cuáles son las fuentes de información para recabar datos. Hoy existe un consenso unánime en considerar que los evangelios canónicos son la fuente principal de información histórica para acceder a Jesús. Pero al mismo tiempo, estos escritos son el mayor problema al cual se enfrenta la crítica historiográfica. Desde Wrede (1901) se ha establecido que podemos encontrar dos tipos de material en los evangelios: el redaccional y el tradicional.

El material redaccional muy posiblemente es creación del evangelista y comprende prácticamente todo el marco cronológico y geográfico que encontramos en las narraciones. Esto quiere decir que los relatos evangélicos no coinciden en esta información, sino que la manipulan, de acuerdo con sus intereses teológicos y planes literarios. Dado el carácter artificial de estos datos, el único material que puede ser usado para una reconstrucción histórica es el tradicional. Sin embargo, estos no son datos históricos puros, sino que han sido compuestos a partir de las necesidades de las comunidades cristianas.

En el material tradicional no solo encontramos recuerdos de la vida y enseñanza de Jesús, sino también aplicaciones de esos recuerdos a la situación de la comunidad cristiana, es decir, se han adherido a estos recuerdos datos que son a su vez redaccionales. Además, encontramos que en la transmisión de la tradición sobre Jesús se han incluido creaciones de los predicadores cristianos, que pretendían seguir con la misión de Jesús. Los cristianos creían que el Señor resucitado seguía hablándoles por medio de la inspiración dada por su Espíritu, por ello los profetas cristianos no tenían reparo en identificar las palabras que ellos pensaban inspiradas como palabras de Jesús, así como identificar las palabras de Jesús como palabras del Señor Resucitado. En este último sentido, las palabras de Jesús adquirieron un cariz totalmente diferente, que las hizo desvincularse de su contexto histórico para asumir un 
significado más amplio, muchas veces de sentido universal o general.

En el material tradicional, por tanto, encontramos: 1) recuerdos objetivos de Jesús (datos anteriores al año 30 d.C), 2) aplicaciones de esos recuerdos en un contexto distinto, así como creaciones de la primitiva comunidad cristiana que se ponen en boca de Jesús (materiales procedentes de entre los años 30 d.C. -70 d.C.), y 3 ) materiales de la época de los evangelistas que reorganizan todo el material tradicional encuadrándolo en un marco cronológico y geográfico artificial (compuestos entre el año 70 d.C. hasta el final de la época de redacción de los textos evangélicos, cerca del 130 d.C.). El problema central de la crítica literaria surge al diferenciar cada tipo de material para definir qué es útil para una reconstrucción histórica. La tarea no es simple, porque al menos el segundo tipo de material fácilmente puede ser confundido con el primero y viceversa, ya que poseen la misma forma o estilo. Por otra parte, el contexto socio-político y religioso era prácticamente el mismo para aquellos cristianos que vivían en Palestina después de la muerte de Jesús, por lo que poco del material cristiano daría signos claros de una creación posterior. La destrucción del templo de Jerusalén y la misión entre los paganos de las ciudades helenísticas por parte de los cristianos, hicieron que el contexto de los evangelistas fuera muy distinto. Por ello, una gran parte de los materiales de esta época puede ser reconocida a partir del contexto que supone. Uno de esos indicios serían las particularidades típicamente helenistas. Sin embargo, no necesariamente todo material con estas características proviene de la diáspora judeocristiana, ya que la expansión del helenismo se había dado ya en toda la cuenca del Mediterráneo. Material con matices o formas helenistas puede ser un auténtico recuerdo de Jesús o una creación suya (Porter, 2000, pp. 126-164).

Los profetas cristianos imitaron el estilo de predicación de Jesús, así que atenernos a la forma de los dichos para determinar su autenticidad es un criterio poco fiable. De la misma manera, tampoco podemos afirmar que la antigüedad de un texto evangélico reflejaría una mayor cercanía con la enseñanza de Jesús o con el recuerdo objetivo de un acontecimiento. Esto porque el proceso de redacción de los evangelios tuvo lugar desde finales de la primera generación cristiana hasta al menos el año 130 d.C. La reinterpretación de los dichos y hechos de Jesús se evidencian en todas las obras.

Aneja a estas cuestiones, nos encontramos con la pregunta acerca de las dependencias literarias entre los evangelios. Si bien 
la teoría de las dos fuentes ${ }^{2}$ es aceptada por la mayoría de los exégetas, su formulación específica varía de autor en autor ${ }^{3}$. Por ejemplo, se discute si Mt. y Lc. conocieron la misma versión de Mc. o de la fuente Q. La misma polémica se da en relación con la transmisión oral de la tradición evangélica, es decir, si el material que utilizaron les llegó como un escrito o por medio de la comunicación oral que en ese tiempo estaba viva. La solución a estas cuestiones no es indiferente a la reconstrucción histórica, porque nos hablaría de la antigüedad de aquello que se ha transmitido y del grado de modificación/creación de la actividad interpretativa de la comunidad cristiana.

2. Esta teoría mantiene que Mc. es el evangelio más antiguo, que sirvió como fuente tanto a Mt. como a Lc. El material discursivo que es común a Mt. y a Lc., pero que no se encuentra en Mc., correspondería a una fuente hipotética llamada Q. Así Mc. y Q. son las fuentes principales para la composición de los otros sinópticos, pero ellas son independientes entre sí. Los materiales propios a Mt. y Lc. son atribuidos a fuentes especiales, M. y L. respectivamente.

3. Para más información general Cf. VargasMachuca, A. (1993). La llamada fuente Q. de los evangelios sinópticos. Teoría de las Dos Fuentes. Modernas Precisiones. Origen, Composición y redacción de la Fuente Q. Su función en el Cristianismo Primitivo. En A. Piñero, Fuentes del Cristianismo. Tradiciones primitivas sobre Jesús (pp. 63-94). Córdoba, Madrid: Alemendro, Universidad Complutense.
En relación con la fuente Q., que propone la teoría de las dos fuentes, se ha asumido que su información es independiente del material marcano. Pero se trata de una propuesta de una fuente totalmente hipotética, nacida para explicar las semejanzas y diferencias de los evangelios sinópticos. Sin embargo, al asumirla como factible, la tarea de su reconstrucción es una cuestión crucial para la determinación del material histórico, porque las versiones mateana y lucana de Q son diversas tanto en palabras, formas verbales o en la extensión de los dichos y/o recuerdos de Jesús. Esto, a su vez, ha llevado a preguntarse si Mt. y Lc. conocieron dos versiones diferentes $\left(Q_{M t}\right.$ y $\left.Q_{L c}\right)$, o si bien existían dos traducciones distintas de un mismo original arameo. En el primer caso, se podría pensar en varias redacciones o reinterpretaciones de Q. a lo largo del tiempo, por lo que el contexto vital de su redacción sería distinto, dando lugar a variadas aplicaciones de la enseñaza de Jesús. En el segundo caso, queda pendiente la búsqueda de un texto original que pueda dar cuenta de las distintas traducciones, tarea que es altamente hipotética y que no omite la posibilidad que en el proceso de traducción se hayan hecho también agregados y cambios al material recibido. 
A este respecto, la aparición del Evangelio Copto de Santo Tomás ha levantado la polémica sobre una tercera versión de Q., que estaría reflejada en este texto $\left(\mathrm{Q}_{\text {EvTom }}\right)$. La relación de esta versión y la fuente Q. a la base de Mt. y Lc. es una cuestión abierta. Mientras algunos piensan que se trata de una versión independiente ${ }^{4}$, la presencia de materiales propios de Mt. y Lc. (M. y L. respectivamente) en el EvTom ha hecho pensar que depende este de los sinópticos, de lo que se desprendería que no estamos delante de una transmisión independiente de $Q^{5}$. Sin embargo, existe la posibilidad que el autor del EvTom conociese por su parte M. y L., si es que se trata de fuentes escritas $u$ orales independientes. La naturaleza del material propio de Mt. y Lc. es muy discutida, porque el estilo propio de cada evangelista se puede notar en ese material, por lo que podríamos considerarlos como creaciones redaccionales de los evangelistas. Estudios sobre la relación de M.y L. con la versión de los mismos dichos en el EvTom pueden resultar útiles, pero no concluyentes, debido

4. Por ejemplo, Koester, H. (2007). From Jesus to The Gospels. Interpreting the New Testament in Its Context. Minneapolis: Fortress Press, pp. 72-83.

5. Así lo mantiene Meier, J. P. (1991). A Marginal Jew. Rethinking The Historical Jesus. (Vol. I). New York, London, Toronto, Sydney, Auckland: Doubleday, pp. 123-139. al carácter fragmentario de este material, que no permite la reconstrucción de una obra con sentido completo. En caso de que hayan sido obras más extensas, se podría pensar que tal vez hayan contenido material que ahora nosotros identificamos como proveniente de Q. Esto explicaría las diferencias en las versiones de esta fuente en los sinópticos. Con todo, esto es altamente hipotético.

Suponiendo la posibilidad de reconstruir Q. desde la base de los testimonios que tenemos de ella, queda el problema de si es posible determinar estratos redaccionales en la fuente. Q. no se escapa de la situación que hemos reseñado más arriba acerca de los materiales tradicionales. Si bien es posible pensar que Q. fue redactada antes del año 70, para Kloppenborg (1999) el material escatológico que contiene ha sido producido por la actividad de los profetas cristianos poco antes o después de la destrucción de Jerusalén y, posteriormente, mezclado con los materiales más antiguos. Con todo, habría que determinar cuál es la procedencia de esos materiales antiguos y su tenor. Este problema tiene mucho que ver con las perspectivas hermenéuticas de los historiadores ${ }^{6}$.

\footnotetext{
6. Una basta colección de artículos al respecto se encuentra en Lindemann, A. (2001). The Sayings Source $Q$ and the Historical Jesus. Leuven: Leuven University Press.
} 
Por ejemplo, ¿hasta qué punto es posible adjudicar a Jesús un pensamiento apocalíptico o al menos escatológico? En el caso de la fuente Q., ¿no es posible pensar que un material que parece directamente vinculado con una explicación escatológica de la destrucción de Jerusalén sea en realidad una reinterpretación de una enseñanza auténtica de Jesús? Otra cosa importante es el grado de actividad literaria que se piensa tuvo la comunidad cristiana y la función que tenía dentro de su vida. ¿Se dio esta para controlar el contenido de la predicación por parte de los profetas cristianos, tal y como lo propone Theissen (2002, pp. 44-52)? Aquí entra en juego la relación que existe entre escrito y transmisión oral.

Un tratamiento aparte debe darse al evangelio de Juan como fuente de conocimiento histórico. $\mathrm{Si}$ bien se puede mantener que su autor conocía otros textos evangélicos, las diferencias entre Jn. y los sinópticos son notorias. Con todo, sus datos no pueden ser considerados inauténticos, aún cuando difieran totalmente de la información que nos dan los otros textos. De hecho, la trama narrativa de Jn. da muchas luces para comprender porqué Jesús entró en un conflicto tan directo con las autoridades judías. La trama narrativa de los Sinópticos resulta demasiado ficcionalizada, en el sentido de que simplifica de tal manera el proceso histórico que resulta inverosímil como secuencia real de acontecimientos que terminara en la muerte de Jesús en cruz. Empero, no hay duda que el texto de Jn es el más teologizado de todos los evangelios. Su fecha de composición, muy posterior a la de los sinópticos, explica los conceptos elevados de su cristología y la aparición de nuevos temas, ausentes en los otros evangelios. Sin embargo, esto no es suficiente para descartarlo como fuente histórica. El problema estriba en determinar qué materiales son antiguos y dignos de consideración. El principal obstáculo en esta empresa es la falta de material paralelo en otros textos que permita una depuración de una posible fuente antigua. En este particular, la investigación de los estratos de la composición joánica continua siendo muy hipotética aunque necesaria desde una perspectiva historiográfica.

La nueva tendencia de estudios sobre el Jesús histórico, conocida como la Third Quest, ha propuesto la utilización de textos extrabíblicos como fuente de información para el Jesús histórico. Ya hemos hablado brevemente sobre la problemática del EvTom. El resto del material evangélico apócrifo tiene en realidad poca relevancia para la investigación acerca de Jesús. En primer lugar porque los relatos apócrifos acerca 
de la infancia están revestidos de un gran ropaje legendario, que claramente proyectan la fe cristiana posterior sobre el primer período de la vida de Jesús, del cual casi no tenemos datos en el N.T. Y, en segundo lugar, porque muchos de los otros evangelios son claramente de tendencia gnóstica y están poco interesados en reseñar acontecimientos reales. Los autores de estos últimos textos usaron la figura de Jesús y sus discípulos como medio de divulgación de sus propias ideas religiosas, por lo que no estaban interesados en dar cuenta de recuerdos objetivos del pasado.

Sin embargo, en los últimos tiempos J. D. Crossan (1992, pp. 462-466) ha suscitado la cuestión de si en el Evangelio de Pedro encontramos una versión independiente de un antiguo relato de la pasión de Jesús. Las opiniones son encontradas, pero hoy nos es imposible reconstruir ese posible texto antiguo, ya que el material está muy entrelazado con motivos alegóricos o legendarios. Por ello, resulta poco útil para la tarea historiográfica. Además el relato de la Pasión tiene un marcado carácter teológico que pretende ofrecer una interpretación religiosa del hecho histórico de la crucifixión de Jesús.

Encontrar otros dichos de Jesús en el material extrabíblico ha sido un proyecto iniciado por $\mathrm{J}$.
Jeremias y proseguido por otros ${ }^{7}$. Pero al máximo podemos comparar el material evangélico con esos otros textos y comprobar su coherencia con las enseñazas de Jesús. Si bien algunos dichos pueden ser mantenidos como auténticos, ofrecen muy poco material nuevo para considerar y que pudiese afectar de manera significativa las consideraciones historiográficas. En efecto, estos dichos solo confirman lo que podemos averiguar a través de la consideración de los textos evangélicos.

No hay duda alguna de que la tarea de reconstruir al Jesús Histórico pasa por la utilización de los textos evangélicos canónicos, que prácticamente son nuestra fuente más confiable. El Jesús de la historia es un producto del análisis de textos que utiliza herramientas historiográficas para evaluar el material a disposición y emitir un juicio sobre su autenticidad. Esta es la realidad de su base metodológica, que define también su limitación y alcance. Un figura histórica de Jesús no puede ir más allá de esta frontera: la producción literaria sobre Jesús jamás agotó ni su

\footnotetext{
7. Recientemente Morrice, W. (2002). Dichos desconocidos de Jesús. Palabras atribuidas a Jesús fuera de los cuatro Evangelios. Santander: Sal Terrae.
} 
recuerdo ni el impacto que causó en quienes lo siguieron, por lo que nuestros esfuerzos solo tendrán un resultado parcial.

\section{Los criterios para determinar la autenticidad de los datos históricos}

La problemática de las fuentes exige articular una serie de criterios para determinar su autenticidad histórica. Pero este es un término equívoco, en el sentido de que no podemos tener certeza total de la autenticidad de los datos; solo podemos hablar de probabilidades y grados de probabilidad en ellos. Eso quiere decir que una información auténtica no puede ser probada como tal por estos criterios, simplemente porque no tiene las características necesarias para ser un material digno de consideración. Esta es una gran limitación, porque al final de cuentas construiremos una figura de Jesús fragmentada e incompleta.

John P. Meier en su obra Jesus. A Marginal Jew (1991, pp. 167-184) ofrece una serie de criterios comúnmente usados para determinar la autenticidad del material. Su anclaje principal es el criterio del testimonio múltiple, aunque afirma que este por sí solo no basta para sacar conclusiones. Sin embargo, la aplicación de estos criterios da como resultado una serie de materiales diseminados, sin un orden claramente definido. Con todo, en su obra la reconstrucción es bastante fluida y rigurosa, sin que por ello se explique el porqué escoge cierto material y desecha otro. La aleatoriedad en la escogencia del material es solo parcial, ya que en este proceso interviene el historiador, quien asume una perspectiva propia a la hora de organizar la información que ha podido recabar.

Precisamente para solucionar el problema de la subjetividad del historiador en la reconstrucción histórica Theissen y Winter (2002) han propuesto que la discusión polémica es el mejor camino para llegar poco a poco a consensos. Pero para que esto tenga lugar es necesario que la propuesta de una figura de Jesús sea plausible para su mundo. Esto significa que tiene que dar cuenta tanto de cómo se inserta en el mundo contextual judío del siglo I d.C, así como explicar los efectos de su persona en el desarrollo del cristianismo primitivo (Theissen \& Merz, 1998). Sanders (2004), por su parte, mantiene que la única forma de ser rigurosos en una propuesta es partiendo de la muerte sufrida por Jesús. Se trata de un acto político que exige una explicación congruente. El problema con esta perspectiva es que otros elementos de la vida de Jesús, como su predicación sapiencial, pueden no ser lo suficientemente considerados, 
porque no tienen una relación directa con su muerte.

En cuanto a la propuesta de Theissen y Winter, se derivan de ella otros problemas importantes, como el estudio y comprensión del contexto socio-histórico. En especial se vuelve fundamental comprender el judaísmo en el que vivió Jesús. Sanders (1992) ha propuesto concentrarse en la reconstrucción del judaísmo generalizado, es decir, aquellas cosas en las que todos los grupos judíos coincidirían, para luego examinar las particularidades sectarias. De esta manera tendríamos un cuadro más completo para colocar a Jesús y a su movimiento como uno de los agentes religiosos en interacción. Pero esto solo ayuda a comprender una parte de la realidad palestina del siglo primero. Malina ${ }^{8}$, Rohrbaugh ${ }^{9}$, Oak$\operatorname{man}^{10}$, Neyrey ${ }^{11}$, Herzog $^{12}$ y otros ${ }^{13}$

8. Malina, B. (2001). The New Testament World. Insigths from Cultural Antropology (3 ed.). Lousville: Westminster John Knox Press.

9. Malina, B., \& Rohrbaugh, R. (1996). Los evangelios sinópticos y la cultura mediterránea del siglo I. Comentario desde las ciencias sociales. Estella, Navarra: Verbo Divino.

10. Oakman, D. (2008). Jesus and the Peasants. Eugene, Oregon: Cascade Books.

11. Neyrey, J. (2005). Honor y vergüenza. Lectura cultural del evangelio de Mateo. Salamanca: Ediciones Sígueme.

12. Herzog, W. (2000). Jesus, Justice, and the Reign of God. A Ministry of Liberation. Lousville: Westminster John Knox Press.

13. Por ejemplo, deSilva, D. (2000). Honor, Patronage, Kinship and Purity. Unlocking New se acercan a los textos desde la ciencias sociales, para comprender los códigos de comunicación e interacción humana, siguiendo las intuiciones de Bailey (1976). Horsley (1996) y otros ${ }^{14}$ se fijan más en la situación política y cómo el Imperio Romano con su poder de dominación afectó a la población, haciendo surgir grupos rebeldes o de resistencia religioso-política.

La pregunta sobre la sociedad en la cual se movió Jesús también pone en evidencia la expansión del helenismo en toda la cuenca mediterránea. Crossan y Reed (2001) deducen desde la evidencia textual y arqueológica algunas características de Jesús. En particular Crossan (1992) se vale de modelos helenistas de comportamiento, como los filósofos cínicos, para proponer un perfil histórico de Jesús. Mientras que otros utilizan modelos sociológicos, como el de los grupos milenaristas, para vincular a Jesús con el movimiento apocalíptico $^{15}$. Se observan, por tanto, dos

Testament Culture. Downers Grove, Illionois: InterVarsity Press; y Stegemann, W., Malina, B., \& Theissen, G. (2002). The social setting of Jesus and the Gospels. Minneapolis: Fortress Press.

14. Por ejemplo, Hanson, K., \& Oakman, D. (1998). Palestine in the Time of Jesus. Social Structures and Social Conflicts. Minneapolis: Fortress Press.

15. Así Ehrman, B. (1999). Jesus. Apocaliptic prophet of the new milennium. New York: Oxford University Press. 
tendencias bien definidas. Una es vincular a Jesús con el sistema cultural más amplio y pluriabarcante, el helenismo, que se entiende como la matriz cultural principal para el época. Mientras que otra insiste en entender el judaísmo de la época como la matriz cultural principal para comprender a Jesús. Esto deja en evidencia que todavía no sabemos bien hasta dónde podemos delimitar una cosa de la otra o cómo integrarlas. Parte del problema es que los testimonios textuales que tenemos han sido escritos en griego y no desde el contexto social en donde se movió Jesús. Además la falta de textos escritos de todas las acciones judías de la época nos condiciona: mucho de lo que sabemos de algunos grupos nos ha llegado por la literatura de adversarios religiosos o sociales, por lo que esta información puede ser muy parcial o inexacta.

¿Hasta qué punto Jesús fue influenciado por el helenismo? ¿Hablaba griego? ¿Predicó en esa lengua? ¿Se conservaron sus propias palabras en los textos del N.T.? En este particular entramos en el campo de las especulaciones, aunque Porter (2000) mantiene que hay evidencia en el N.T. de situaciones en las que Jesús seguramente habló en griego. Anejo a esta cuestión encontramos el problema de determinar la relación que Jesús tenía con las ciudades helenísticas de Galilea y su procedencia rural. Los evangelios no dan cuenta de una presencia de Jesús en esos sitios, pero eso no indica que no haya predicado realmente en ellas o que no haya tenido relación con la vida urbana antes de iniciar su ministerio público. Qué tanto Jerusalén puede ser entendida como una polis griega, no deja de ser una pregunta significativa para aproximarnos a Jesús y a sus acciones en ella.

Como podemos ver, algunos de los criterios que nos permiten enmarcar a Jesús dentro de su contexto cultural parten de precomprensiones de la situación palestina en el contexto del Imperio Romano. El estudio de los textos de la época del judaísmo helenístico, así como otras obras contemporáneas, nos permiten tener un panorama más amplio de este complejo mundo cultural ${ }^{16}$. Sin embargo, los textos literarios no necesariamente reflejan la cultura popular en la que se manejaban Jesús y sus discípulos, como tampoco agotan todas las aristas del fenómeno cultural. Por eso se ha hecho necesario vincular el estudio de los escritos con los testimonios arqueológicos. En este punto hemos podido constatar que no necesariamente lo que nosotros leemos en la literatura

16. Véase Zeitlin, I. (1994). Jesus and the Judaism of his Time. Cambridge: Polity Press. 
tiene su contraparte en la realidad arqueológica. Un caso paradójico lo tenemos con los grupos esenios. A nivel literario los textos de autores externos a este grupo parecen contradecirse en sus afirmaciones, además que las primeras hipótesis referidas a Qumrán han dejado una huella difícil de borrar que muchas veces ha sido puesta en cuestión por los arqueólogos. El grupo esenio parece estar vinculado con los primeros cristianos, pero de qué manera lo estuvo o qué relación podía tener Jesús con ellos es algo que todavía se discute y que resulta altamente hipotético ${ }^{17}$.

Por otra parte, la evolución del judaísmo después de la caída de Jerusalén en el año 70 d.C ha dejado una huella importante en la literatura judía posterior, que releyó la historia pasada desde perspectivas nuevas, pero vinculadas con ideologías religiosas ya existentes en tiempos de Jesús. Esto hace difícil separar los estratos ideológicos de las fuentes. Los criterios que tenemos para hacerlo obedecen más a posiciones previas de los historiadores que a realidades objetivas, por lo que la polémica es encendida. Parece ser que el debate y la continua revisión de los resultados

17. Véase la obra de Taylor, J. (2003). ¿De dónde viene el cristianismo? Estella, Navarra: Verbo Divino. es la única vía para clarificar y corregir posturas. Sobre todo es importante la revisión de los métodos histórico-críticos y la utilización de otros métodos de análisis social o cultural.

\section{Condicionamientos hermenéuticos de los historiadores}

En su interesante libro The hermeneutical Jesus, Stewart (2008), ha dejado en evidencia que en el fondo de la investigación histórica sobre Jesús se encuentran las opciones interpretativas de los autores. Fernando Bermejo (2006) aduce, por ejemplo, que la investigación sobre Jesús ha alcanzado ya su límite y que los diferentes matices que se observan en las obras de los investigadores creyentes no son más que un intento desesperado de salvar su figura de los tajantes resultados de Reimarus, de Baur o de Scweitzer. En otras palabras, negarse a aceptar que Jesús fue un líder revolucionario judío que fracasó en su intento de obtener el poder político en Jerusalén, es solo una quimera nacida del ansia de probar la verdad de la fe cristiana. En esta posición tan radical también vemos una posición filosófica: toda la teología cristiana no es más que un invención posterior que poco o nada tiene que ver con el Jesús histórico. 
Este es el dilema de toda hipótesis en relación con Jesús: ¿cómo explicar su figura histórica con el nacimiento de un cuerpo literario tan extenso pero marcado por claros motivos religiosos? La relación entre historia y religión en el caso de Jesús no es meramente circunstancial. La época en la cual Jesús vivió estaba marcada por una clara relación entre la vida personal y social con los paradigmas de comprensión religiosa. Toda la vida social estaba determinada por esa relación, fuese en el ámbito doméstico como en el político. Podríamos afirmar, entonces, que es posible realizar un doble acercamiento a la persona de Jesús. Por un lado, podríamos tratar de entender su influencia socio-política, ya que al ser un líder religioso necesariamente su actividad pública tendría directa relación con la continuidad o discontinuidad del statu quo. Por otro, podríamos considerar su actividad muerte como producto de un conflicto con los líderes religiosos de la época, ya que Jesús se opondría a sus postulados religiosos. Este conflicto degeneró en una resolución política, como un medio de represión.

Ambas posibilidades son válidas, pero sus resultados pueden ser abiertamente disímiles. Por ejemplo, Wright (1996) plantea la posibilidad de reconstruir una posible autocomprensión de Jesús analizando las ideas religiosas en boga. Con su método pretende encontrar una base ideológica lo suficientemente clara como para explicar el porqué de sus opciones y predicación. Sería desde esta misma base, común a una gran parte de los judíos contemporáneos suyos, que los primeros discípulos y luego los evangelistas habrían construido el contenido de la antigua predicación cristiana y desde ella los escritos del N.T. Esta realidad permitió la suficiente apertura ideológica para que surgieran diversas posiciones teológicas, que son típicas de los escritos neotestamentarios. Sin embargo, su unidad sería garantizada por su referencia a Jesús y su pensamiento teológico. No hay duda de que el argumento es correcto: es imposible entender el contenido de los textos del NT y de las interpretaciones que estos hacen de la persona de Jesús, si no es a través de una comprensión suficiente de las concepciones religiosas subyacentes en la época.

Por otra parte, una visión eminentemente política de Jesús, tal y como la propone Bermejo, puede resultar insuficiente para explicar el porqué los cristianos crearon un sistema religioso tan homogéneo después de su muerte. Si Jesús no tenía claras ideas religiosas que pudieran facilitar el surgimiento de interpretaciones teológicas acerca de él, todo quedaría sujeto a la inventiva 
de las comunidades cristianas -tal y como lo proponía Bultmann- lo cual es inverosímil. Mucha razón tiene Gonzalo Puente Ojea (2008) al afirmar que la existencia de Jesús se ve comprobada en la gran variedad de interpretaciones que surgieron de su persona, pero esto también nos hace pensar que el efecto de actividad pública sobre todo se centró en el cuestionamiento de las comprensiones religiosas mantenidas por las autoridades del Templo de Jerusalén.

En efecto, la gran variedad de corrientes religiosas en tiempos de Jesús y las disputas teológicas entre ellas no crearon grandes polaridades que llegaron a convertirse en conflictos sociales de gran magnitud. Por eso, la visión que presentan los evangelios acerca de la cooperación entre fariseos y los sacerdotes del Templo, parece ser una retroproyección de las tensiones entre los cristianos de la segunda generación con el grupo fariseo, más que una realidad histórica de tiempos de Jesús. Si es así, el conflicto que terminó en la muerte de Jesús tuvo que estar protagonizado por aquellos que tenían tanto un poder político como religioso. En este particular, la visión de Sanders (2000) acerca de la muerte de Jesús es cierta: estamos delante de una acción política por parte de los sacerdotes y de los romanos surgida como respuesta a opciones religiosas de Jesús. Opciones que claramente ponían en cuestión la autoridad de estas instancias sociales en virtud de un poder superior. Permanece, sin embargo, una cuestión central: ¿Tenía Jesús la pretensión de ser el Mesías?

Aquí de nuevo entramos en un problema hermenéutico de primer orden, sobre todo al constatar que las ideas mesiánicas no eran uniformes en tiempos de Jesús. Esto nos empuja a considerar cuáles podrían ser las opiniones de Jesús al respecto. En este particular ha adquirido especial relieve la figura del hijo del hombre, que en los evangelios solo es puesta en labios de Jesús. La extraña coincidencia de significados distintos en los dichos de Jesús ha levantado la cuestión de si es un título usado por él para explicar su identidad, o si se refería a una figura escatológica distinta de su persona, o si bien es una creación cristiana pospascual. La ambivalencia del concepto en los textos evangélicos es tal que ninguna solución ha creado consenso. El estudio de otras corrientes de pensamiento judío, sobre todo las reflejadas en los apócrifos del AT, podrían traer más luz en el futuro ${ }^{18}$. Pero así

18. Estudios sobre el tema se pueden encontrar en Boccaccini, G. (2007). Enoch and the Messiah Son of Man. Revisiting the Book of the Parables. Grand Rapids, Cambridge: William B. Eerdmans Publishing Company. 
como no se tiene claridad en lo que este símbolo quiere significar, tampoco la tenemos en cuanto a las pretensiones mesiánicas de Jesús.

Desde este punto de vista podemos acercarnos al problema de la actividad de Jesús como predicador. Algunos pensadores como Borg ${ }^{19}$, Crossan y el Jesus Seminar, han propuesto la imagen de Jesús como un maestro de sabiduría. Siguiendo distintos métodos, se subrayan unas particularidades del pensamiento de Jesús como un predicador de una forma de vida buena, pero coinciden en mantenerlo alejado de un movimiento escatológico o apocalíptico. Jesús promovería un nuevo estilo de vida basado en una crítica a las estructuras culturales dominantes del honor-vergüenza y el patriarcalismo. Es desde esta visión ética de la vida donde adquiere sentido la propuesta de Jesús y los símbolos que usa en su predicación. Sin embargo, no parece lógico suponer que una actividad como esta haya suscitado una oposición tan fuerte dentro del ámbito político-religioso. Si bien la crítica a los paradigmas sociales más tradicionales sería un

19. Borg, M. (1995). Meeting Jesus again for the First Time. New York: Haper Collins Publishers; y (2008). Jesus. Uncovering the Life, Teachings, and Relevance of a Religious Revolutionary. New York: HarperOne. programa contracultural, esta no pone en vilo la permanencia del poder político y su plataforma ideológica. Se podría entrever que dicha actividad hubiera gozado de poca popularidad, por lo que la base social que la apoyaría no sería lo suficientemente grande como para haber preocupado a las autoridades en Jerusalén.

Visto desde aquí, el conflicto creciente de Jesús narrado por Jn. en las fiestas judías, puede vislumbrarse como un recuerdo histórico auténtico. Es cierto que los diálogos polémicos de Jesús con sus adversarios judíos están marcados por claros desarrollos cristianos. La alta cristología joánica se hace evidente en todas las discusiones para resaltar la autoridad indiscutida de Jesús. Pero eso no obsta para que sea posible proponer la existencia de estratos antiguos de la tradición, que fueron preservados por el evangelista. Esta confrontación directa con el Templo no era extraña en otros grupos judíos contemporáneos, pero supone también un buena cuota de conceptos escatológicos, que servían como medio de crítica y de propuestas de superación del sistema religioso imperante. Un ejemplo elocuente lo tenemos en los textos escatológicos de Qumrán. Pero el pensamiento escatológico judío no era uniforme, ni en sus símbolos ni en sus concepciones acerca del fin de 
la historia. El reto estriba, por tanto, en tratar de definir cuál era el pensamiento de Jesús al respecto desde criterios más objetivos. Este reto aún persiste en la investigación sobre su predicación.

\section{El Jesús histórico y la reflexión cristológica: ¿Algún punto en común?}

Hablar del Jesús histórico no es referirse al Cristo de la fe. Así como hablar del Cristo de la fe no implica excluir a la figura histórica de Jesús. La primera afirmación se puede entender mejor cuando nos percatamos de que la reconstrucción histórica de Jesús empezó en un ámbito no eclesial y bajo los presupuestos de la Ilustración (Fusco, 1999), aunque desde entonces se convirtió en un desafío para la fe cristiana. Se partía de la distinción entre la fe y la historia, como si fueran dos realidades totalmente separadas, pero en donde la primera había desfigurado totalmente la segunda. En otras palabras, las obras cristianas habían construido una imagen de Jesús totalmente nueva, que ocultaba a la verdadera persona histórica detrás de un ropaje religioso. Por ello, era necesario librar a Jesús de todo aquello que los cristianos habían creado. En el fondo existía un distanciamiento de la fe cristiana y la historia: ambas eran totalmente independientes, los cristianos no estaban interesados en el Jesús real, solo en su construcción cristológica.

Fusco advierte en Schweitzer un falta de distinción entre el "Jesús terreno" y el "Jesús historiográfico", que en la terminología de Meier podríamos llamar el "Jesús real" y el "Jesús de la historia". En efecto, el N.T establece una clara identificación entre el Jesús real y el Cristo de la fe. Desde este punto de vista cabe preguntarse si los textos están interesados o no en el "Jesús historiográfico". Es claro que este último no es más que una construcción teórica, basada en los principios de la probabilidad y el análisis de los datos a disposición. Pero el claro interés de identificar al personaje real con la confesión de la fe indica que para los primitivos cristianos resultaba imposible separarlos: la fe tiene sentido solo y en función de la persona a la cual se confiesa como Kyrios. Para nada tendría sentido la investigación historiográfica de Jesús para ellos, ya que esta es solo una caricatura de lo que ellos consideraban la auténtica persona de Jesús.

En otras palabras, la cristología neotestamentaria quiso asentarse en el recuerdo objetivo de Jesús, aunque interpretándolo para dar cuenta de la profundidad de su misterio y de su presencia como Señor resucitado en la vida misma de la Iglesia. ¿Puede tener alguna 
utilidad para la cristología actual la investigación sobre el Jesús histórico? Sin duda alguna, porque nos permite comprender los procesos que van desde lo acontecido hasta lo interpretado; desde lo vivido hasta lo recordado como significativo en el evangelios y narrado como un camino de crecimiento en la fe. Entre la cristología del NT y el Jesús real no se quiso establecer un abismo de artificialidad, sino una continuidad en la profundización de la significación. Pero la tarea histórica tiene un sentido más limitado, puesto que se basa en lo probable, no en la significación humana de la figura histórica.

Hoy propongo construir una nueva cristología desde los resultados de la investigación histórica acerca de Jesús. Se suscita, por tanto, una pregunta: ¿En qué consiste este proyecto? Si con él se entiende el prescindir de la cristología del N.T para elaborar una nueva, se llega a la conclusión de que los parámetro conceptuales del N.T resultan insuficientes para comprender el misterio del Cristo manifestado en Jesús. Pero esto nos pone en una situación equívoca, porque a nivel historiográfico podemos obviar una serie de recuerdos objetivos que no resultan ser probables, por lo que la imagen histórica de Jesús se empobrece. Desde estos datos la reflexión cristológica resulta carente, porque nos basamos en una abstracción que puede ser manipulada desde los condicionamientos hermenéuticos propios de los historiadores. En este sentido, el quehacer cristológico se transforma en una acción política, no raras veces en franco conflicto con el desarrollo dogmático posterior. Evocar la figura histórica de Jesús para imponer una nueva "ortodoxia" teológica implica la voluntad de construir una nueva organización eclesial, que en principio no significa desear algo negativo, pero que sí utiliza inconvenientemente una investigación que tiene alcances limitados, usándola como soporte ideológico.

Por otro lado, usando la información recabada por el método histórico-crítico, podemos entrar en el mundo de la construcción de la teología neotestamentaria. Sin embargo, aquí también el conocimiento acerca del Jesús histórico tiene que ser complementado con un estudio acerca de la evolución del cristianismo primitivo y su situación socio-histórica. Por ello no es extraño que muchos de los investigadores del Jesús histórico hayan proseguido su obra describiendo este proceso. Esto deja en evidencia que la vida y predicación de Jesús han dejado una profunda huella en sus seguidores. La referencia obligada de todo cristiano a los evangelios así lo muestra. No es raro que eminentes estudiosos judíos 
también se hayan abocado a la investigación de Jesús y sus seguidores posteriores como parte del desarrollo de las corrientes judías en la época helenística. Se concluye, por tanto, que separar al Jesús histórico de los desarrollos teológicos del NT y, por consiguiente, de la vida de la comunidad cristiana primitiva supone desvirtuar los efectos que el Jesús real suscitó en sus seguidores.

\section{Bibliografía}

Bailey, K. (1976). Poet and Peasant. Grand Rapids: Eerdmans Publishing Company.

Bermejo, F. (2005). Historiografía, exégesis e ideología. La ficción contemporánea de las "Tres búsquedas" del Jesús Histórico (I). (F. d. Catalunya, \& F. d., Edits.) RcaT , $X X X(2), 349-406$.

Bermejo, F. (2006). Historiografía, Exégesis e Ideología. La ficción contemporánea de las "Tres Búsquedas" del Jesús Histórico (y II). RCatT , XXXI (1), 53-114.

Boccaccini, G. (2007). Enoch and the Messiah Son of Man. Revisiting the Book of the Parables. Grand Rapids, Cambridge: William B. Eerdmans Publishing Company.

Borg, M. (2008). Jesus. Uncovering the Life, Teachings, and Relevance of a Religious Revolutionary. New York: HarperOne.

Borg, M. (1995). Meeting Jesus again for the First Time. New York: HaperCollins Publishers.

Crossan, J. D. (1992). The Historical Jesus. The Life of a Mediterranean
Jewish Peasant. New York: HarperSanFrancisco.

Crossan, J. D., \& Reed, J. (2001). Excavating Jesus. Beneath the stones, behind the texts. New York: HarperSanFrancisco.

deSilva, D. (2000). Honor, Patronage, Kinship and Purity. Unlocking New Testament Culture. Downers Grove, Illionois: InterVarsity Press.

Ehrman, B. (1999). Jesus. Apocaliptic prophet of the new milennium. New York: Oxford University Press.

Fusco, V. (1999 8-Mayo). Pasato e futuro nella "Ricerca del Gesù Storico". Accesado el 21-setiembre de 2010 desde el Pontificium Institum Biublicum de Urbe: http://www.biblico.it/doc-vari/ conferenza_fusco.html

Hanson, K., \& Oakman, D. (1998). Palestine in the Time of Jesus. Social Structures and Social Conflicts. Minneapolis: Fortress Press.

Herzog, W. (2000). Jesus, Justice, and the Reign of God. A Ministry of Liberation. Lousville: Westminster John Knox Press.

Horsley, R. (1996). Archeology, History and Society in Galilee. The social context of Jesus and the Rabbis. Harrisburg, Pennsylvania: Trinity Press International.

Kloppenborg, J. S. (1999). The Formation of Q. Trajectories in Ancient Wisdom Collections. Harrisburg: Trinity Press International.

Koester, H. (2007). From Jesus to The Gospels. Interpreting the New Testament in Its Context. Minneapolis: Fortress Press.

Lindemann, A. (2001). The Sayings Source $Q$ and the Historical Jesus. Leuven: Leuven University Press. 
Malina, B. (2001). The New Testament World. Insigths from Cultural Antropology (3 ed.). Lousville: Westminster John Knox Press.

Malina, B., \& Rohrbaugh, R. (1996). Los evangelios sinópticos y la cultura mediterránea del siglo I. Comentario desde las ciencias sociales. Estella, Navarra: Verbo Divino.

Meier, J. P. (1991). A Marginal Jew. Rethinking The Historical Jesus. (Vol. I). New York, London, Toronto, Sydney, Auckland: Doubleday.

Morrice, W. (2002). Dichos desconocidos de Jesús. Palabras atribuidas a Jesús fuera de los cuatro Evangelios. Santander: Sal Terrae.

Neyrey, J. (2005). Honor y vergüenza. Lectura cultural del evangelio de Mateo. Salamanca: Ediciones Sígueme.

Oakman, D. (2008). Jesus and the Peasants. Eugene, Oregon: Cascade Books.

Porter, S. (2000). The Criteria for Authenticity in Historical-Jesus Research. Previus Discussion and New Proposals. London, New York: T\&T Clark International.

Puente Ojea, G. (2008). La existencia histórica de Jesús en las fuentes cristianas y su contexto judío. En A. Piñero, ¿Existió Jesús realmente? (págs. 167-199). Madrid: Raíces.

Sanders, E. (2004). Jesús y el Judaísmo. Madrid: Trotta.

Sanders, E. (1992). Judaism.: Practice and Belief. 63 BCE-66 CE. London, Philadelphia: SCM Press, Trinity Press International.

Sanders, E. (2000). La figura histórica de Jesús. Estella, Navarra: Verbo Divino.
Stegemann, W., Malina, B., \& Theissen, G. (2002). The social setting of Jesus and the Gospels. Minneapolis: Fortress Press.

Stewart, R. (2008). The Quest of the Hermeneutical Jesus. The impact of Hermeneutics on the Jesus Research of John Dominic Crossan and N. T. Wright. Lanham, Boulder, New York, Toronto, Plymouth: University Press of America.

Taylor, J. (2003). ¿De dónde viene el cristianismo? Estella, Navarra: Verbo Divino.

Theissen, G. (2002). La redacción de los evangelios y la política eclesial. Un enfoque socio retórico. Estella, Navarra: Verbo Divino.

Theissen, G., \& Merz, A. (1998). The Historical Jesus. A Comprehensive Guide. Minneapolis: Fortress Press.

Theissen, G., \& Winter, D. (2002). The Quest for The Plausible Jesus. The Question of Criteria. Lousville, London: Westminster John Knox Press.

Vargas-Machuca, A. (1993). La llamada fuente $\mathrm{Q}$ de los evangelios sinópticos. Teoría de las Dos Fuentes. Modernas Precisiones. Origen, Composición y redacción de la Fuente $\mathrm{Q}$. Su función en el Cristianismo Primitivo. En A. Piñero, Fuentes del Cristianismo. Tradiciones primitivas sobre Jesús (págs. 63-94). Córdoba, Madrid: Alemendro, Universidad Complutense.

Wrede, W. (1901). Das Messiasgehemnis in den Evangeliem. Zugleich ein Beitrag zur Verstädnis des Markusevangeliums. Gotinga, Alemania.

Wright, N. (1996). Jesus and the Victory of God. Minneapolis: Fortress Press.

Zeitlin, I. (1994). Jesus and the Judaism of his Time. Cambridge: Polity Press. 
